\title{
Apps, mHealth y telemedicina: beneficios, peligros, paradigmas y nuevas fronteras en medicina
}

\author{
Apps, $m$ Health and telemedicine: benefits, dangers, paradigms and new frontiers in medicine
}

\author{
Aland Bisso-Andrade'
}

Bisso-Andrade A. Apps, mHealth y telemedicina: beneficios, peligros, paradigmas y nuevas fronteras en medicina. Rev Soc Peru Med Interna. 2019;32(3):87-88. https://doi.org//0.36393/spmi.v32i3.473
El uso de la tecnología móvil en el campo la de salud tiene el potencial de transformar las prestaciones de servicio de salud en niveles insospechados. La Organización Mundial de la Salud (OMS) la denomina 'sanidad móvil' y la define como "la práctica de la medicina y la prestación de servicios sanitarios mediante dispositivos móviles, como teléfonos móviles, dispositivos de seguimiento de pacientes, asistentes digitales personales (PDA, en sus siglas en inglés) y otros dispositivos inalámbricos". ${ }^{1}$ La abreviatura de 'salud móvil' es mHealth, un término que se utiliza para referirse a la práctica de la medicina y a la salud pública con el apoyo de dispositivos móviles. ${ }^{1}$

El servicio de salud, además de tener calidad, debe ser oportuno. Los profesionales de la salud, así como todo aquel que alguna vez requirió una atención médica, saben que la distancia, las limitantes geográficas y la insuficiente oferta de centros médicos constituyen un factor negativo que muchas veces ha generado serias complicaciones, incluso la muerte en el paciente que no recibió una atención adecuada a tiempo. En este sentido, la actual tecnología de las comunicaciones puede brindar herramientas valiosas que facilitarían la conexión inmediata entre un paciente y un profesional de la salud. En un pueblo alejado, puede faltar un médico, pero de seguro podemos encontrar una computadora conectada a Internet y muchos teléfonos móviles. La expansión de los llamados teléfonos inteligentes, así como la de las redes 3G y 4G, ha impulsado la utilización de aplicaciones móviles (Apps) que ofrecen diversos servicios de atención sanitaria. Además, la tecnología permite hoy en día que los pacientes puedan conectarse a diversos dispositivos médicos, o sensores, bajo forma de brazaletes o relojes, a fin de enviar valiosa información a distancia, y en tiempo real, sobre sus funciones vitales, variables bioquímicas, electrocardiográficas, entre otras. Así mismo, muchas Apps de mHealth brindan información sanitaria y orientación psicológica y del estilo de vida, recordatorios de toma de medicación, consultas médicas en línea y, en general, una innumerable serie de

I. Médico internista, Clínica Delgado. Past presidente de la SPMI servicios destinados a cubrir todas las necesidades de salud. Sin embargo, no todo lo tecnológico es bueno. A la fecha, se considera la existencia de unos 325000 Apps de salud disponibles, principalmente, en las plataformas de Google Play Store y de Apple Play Store. Los ámbitos con mayor desarrollo son conexión con el doctor (30 \%), diabetes (27\%), corazón y sistema circulatorio (24\%), adherencia a la medicación (24\%), hábitos saludables y fitness $(22,5 \%)$, eficiencia hospitalaria (19\%) y salud mental (17\%). Solo en el año 2017 se calculó un número de 370 millones de descargas. ${ }^{2,3}$ Por tanto, la información que se intercambia responde a innumerables variables y es por demás heterogénea, de modo que no se puede asegurar que las Apps confieran siempre un margen de error en niveles no significativos. Principalmente, aquellas que envían información de datos clínicos o los referidos a consultas médicas online, en la cual un profesional de la salud al otro lado de la pantalla solo podrá escuchar y ver al paciente, pero sin posibilidad de realizar una historia semiológica detallada y menos un examen físico. En este caso, el riesgo de error para sacar conclusiones y emitir una recomendación o prescripción médica acertada se incrementa. Sabemos que la información y la comunicación en salud, desprovistas de los filtros necesarios, sin la normativa adecuada y sueltas al libre albedrío, enarbolan un peligro potencial de alcances insospechados. Es necesario que aspectos básicos, como la seguridad, la confidencialidad, la autonomía y la calidad, estén plenamente garantizados, por tanto, es necesario que los médicos y las autoridades sanitarias de cada país tengan un rol activo a fin de garantizar que todos los alcances de la telemedicina sean seguros y justos para toda la población. ${ }^{4}$

Los desarrolladores de Apps saben que el mercado de la salud es muy grande, de ahí que su notable incremento responde a objetivos financieros claramente apetecibles. Esta situación ha determinado la puesta en práctica de normativas y de la exigencia de certificación de aplicaciones. En Estados Unidos, la Food and Drugs Administration (FDA) acaba de actualizar su documento de 'Política para funciones de software de las aplicaciones médicas móviles' a fin de dar los lineamientos de 
las funciones, seguridad, limitaciones, facturación, entre otras características, de todas las Apps relacionadas a la salud. ${ }^{5}$

Según la FDA, si una aplicación móvil está destinada a ser utilizada en la realización de una función de dispositivo médico; es decir, si se utiliza para el diagnóstico de una enfermedad, o la curación, mitigación, tratamiento o prevención de enfermedad, se considera un dispositivo médico, independientemente de la plataforma en la que se ejecuta. Sobre la salud móvil, la Comisión Europea publicó en 2014, en Bruselas, el Libro Verde sobre Salud Móvil en la UE, donde se manifiestan que los sistemas sanitarios de Europa se enfrentan a nuevos desafíos, tales como el envejecimiento de la población y el aumento de la presión presupuestaria y que, en este contexto, la sanidad móvil podría ser una de las herramientas para abordar estos retos y contribuir al desarrollo de una atención sanitaria más centrada en el paciente y apoyo al cambio hacia la prevención, al tiempo que se mejora la eficacia del sistema. ${ }^{6} \mathrm{El}$ documento establece algunas recomendaciones en torno a la protección de datos, la transparencia de la información, el marco jurídico y las responsabilidades derivadas en torno a la seguridad de las Apps. Además, recomienda que los programas de certificación también puedan ser indicadores fiables para los profesionales de la salud y el público usuario, ya que podrían verificar si el App ofrece contenido veraz y tiene garantías respecto a la seguridad de los datos de los usuarios.

En el Perú, la Ley Marco de Telesalud (Ley N. 30421 ) tiene el objeto de establecer los lineamientos generales para la implementación y el desarrollo de la tecnología de la información y la comunicación para el servicio de salud a distancia, principalmente a los usuarios en áreas rurales o con limitada capacidad resolutiva. Esta Ley tiene como ámbito tanto los servicios de salud públicos como privados, y establece que el Ministerio de Salud, en su calidad de ente rector del sector Salud, formula, planea, dirige, coordina, ejecuta, supervisa y evalúa la política nacional y sectorial de telesalud. ${ }^{7}$

En agosto de 2019, en el Perú circuló por las redes sociales un vídeo que le hacía propaganda a un App perteneciente a una empresa de seguro privado en el cual se ofrecían consultas médicas a través de un videochat. En apariencia, esta herramienta es para que un paciente "pueda hacer una consulta sin salir de casa y sin que el médico vaya a la suya...y todo desde tu celular", tal como lo anuncia el médico que aparece en el vídeo. Es evidente que el objetivo fundamental del seguro privado es financiero, no se necesita ser un experto para concluir que a los seguros les conviene disminuir la carga de consultas médicas directas en clínicas privadas a fin de ahorrarse el pago de honorarios médicos y otros costos que la consulta pueda generar. Sin embargo, más allá de este aspecto, más grave es el riesgo de salud que implica hacer una consulta médica no presencial debido a que la falta de un examen clínico directo puede ocasionar la ausencia de información valiosa y, en consecuencia, derivar en una recomendación médica errónea. Un dolor abdominal consultado por un App podría deberse a un abdomen agudo quirúrgico, una pancreatitis, incluso hasta una neumonía basal o un infarto de miocardio, entidades que cuando son de presentación atípica pueden ser banalizadas o pasar desapercibidas si la consulta no incluye un buen examen físico. Hace muchos años que ya existe en todo el mundo las Apps para hacer consultas en línea, pero los límites deben estar normados y debidamente supervisados. De hecho, son útiles cuando se utilizan para dar una orientación o consejo médico general, pero si son para sacar conclusiones diagnósticas e, incluso, generar una receta farmacológica, el delgado hilo de seguridad se podría romper y las consecuencias pueden ser gravísimas. De otro lado, la adecuada relación médico-paciente se construye a partir de la consulta directa, sea domiciliaria o en un consultorio. Una App no lo puede reemplazar. Mis pacientes se sienten mejor cuando nos damos un apretón de manos, conversamos en detalle y perciben que a través del tacto y mi estetoscopio los examino con detenimiento. A través del vídeo de un celular no es posible palpar un ganglio, ni siquiera usar un bajalenguas.

\section{REFERENCIAS BIBLIOGRÁFICAS}

I. Organización Mundial de la Salud, mHealth - New horizons for health through mobile technologies, Global Observatory for eHealth series 20II; volumen 3; p.6. URL: https://www.who.int/goe/publications/goe_ mhealth_web.pdf (Visto el 3-10-2019)

2. Fundación Isys. Informe anual sobre el mercado de Apps de salud de Research2Guidance. URL disponible en: https://www.fundacionisys. org/es/blogs/mhealth/3 I2-informe-anual-sobre-el-mercado-de-apps-desalud-de-research2guidance (Visto el 3-10-2019)

3. mHealth Economics 2017. Current status and future trends in mobile health. URL disponible en: https://research2guidance.com/product/ mhealth-economics-20I7-current-status-and-future-trends-in-mobilehealth/ (Visto el 03-10-2019)

4. Sim I. Mobile devices and health. New Eng J Med. 20I9;38I(I0):956-968.

5. US. Food and Drugs Administration. Policy for device software functions and mobile medical applications. Document issued on September 27, 2019. URL disponible en: file://C:/Users/DELUCONSULT419/ Downloads/FDA\%20telemedicina.pdf (Visto el 03-10-2019)

6. Libro Verde sobre Sanidad Móvil. Comisión Europea. Bruselas 10.04.2014(COM2014)219Final. URL disponible en: https:/lec.europa. eu/transparency/regdoc/rep/I/20 I4/ES/I-20 I4-2I9-ES-FI-I.Pdf (Visto el 04-10-2019)

7. Ley Marco de Telesalud (Ley N. ${ }^{\circ}$ 3042I). Publicada en El Peruano, el sábado 2 de abril de 2016. 